\title{
Autosomal recessive spastic paraplegia type 69
}

INSERM

\section{Source}

INSERM. (1999). Orphanet: an online rare disease and orphan drug data base. Autosomal recessive spastic paraplegia type 69. ORPHA:401830

Autosomal recessive spastic paraplegia type 69 is a rare, complex hereditary spastic paraplegia disorder characterized by infantile onset of progressive lower limb spasticity, global developmental delay, hyperreflexia, clonus and extensor plantar reflexes, associated with dysarthria, intellectual disability, cataracts and hearing impairment. 